\title{
The Use of Augmented and Mixed Reality Technology to Improve Surgical Outcomes: a Systematic Review Protocol
}

Edward Bollen ( $\nabla$ edward.bollen.15@ucl.ac.uk)

University College London Medical School https://orcid.org/0000-0002-9841-2219

Joshua Solomon

Royal Free Hospital

Matthew Stubbs

Royal Free Hospital

Benjamin Langridge

Royal Free Hospital

Peter E. Butler

Royal Free Hospital

\section{Protocol}

Keywords: Augmented reality, Mixed reality, Surgery, Intraoperative, Safety, Systematic review

Posted Date: December 10th, 2020

DOI: https://doi.org/10.21203/rs.3.rs-123463/v1

License: (c) (i) This work is licensed under a Creative Commons Attribution 4.0 International License. Read Full License 


\section{Abstract}

\section{Background}

The use of mixed and augmented reality in surgery is a novel and rapidly developing field. Augmented reality is a technology which overlays virtual images on to the real world and is supported by a variety of platforms. Mixed reality is a newer technology, which, in contrast, allows the user to interact with virtual objects projected onto the real world. These technologies have been employed in a range of surgical contexts and specialities and have generated significant public interest, however, the evidence supporting their proposed benefits is unclear. This systematic review aims to critically appraise the intraoperative use of mixed and augmented reality technologies to improve surgical outcomes and provide directions for future research.

\section{Methods}

This systematic review will follow the PRISMA guidelines and search the MEDLINE, EMBASE, the Cochrane Library electronic databases. All clinical studies reporting empirical data on the intraoperative use of augmented or mixed reality technologies will be eligible for inclusion. Identified studies will be screened for inclusion by three authors in parallel, with a fourth author resolving any discrepancies. Risk of bias will be assessed in accordance with Cochrane Handbook for Systematic Reviews of Interventions guidance. The quality of evidence for each outcome will be assessed using the GRADE approach.

\section{Discussion}

This will be the first systematic review performed with a specific focus on the use of mixed reality in surgery. If augmented and mixed reality are to become established tools in surgery, it is necessary to understand their use cases, advantages, limitations and cost-effectiveness. This is a young but rapidly evolving field; this synthesis of the current evidence base will provide direction for future research and development.

Systematic Review Registration

PROSPERO CRD42020205892

\section{Background}

Surgery is a field of constant innovation, driven by the objective of optimising patient outcomes. Technological advancements frequently provide exciting opportunities for such innovation but frequently a gap exists between the hype surrounding a technology and its initial capabilities. Technological advances are helping to drive the digitalisation of modern healthcare, a phenomenon which has never been more obvious than during the coronavirus disease 2019 (COVID-19) pandemic. Notably, augmented reality (AR) has gained much attention in this time for its use in telemedicine platforms, facilitating remote surgical collaboration on a global scale. AR is a form of extended reality technology, alongside mixed reality (MR) and virtual reality (VR), which provides the user with an altered perception of their surroundings. A surgeon may attend a live operating theatre on the other side of the world using AR, see internal anatomy superimposed on a patient using MR or practise procedural skills on virtual patients using VR. At the heart of each use is the drive to improve patient care and safety.

The use of AR allows the individual to visualise virtual images overlaid on to the real world. The basic components of an AR system are a camera, a processor and a display which, respectively, record a real-world image, combine this with a virtual image, and present the combined image to the user. In the context of surgery, the virtual image may be, for example, a 2-dimensional radiograph, or 3-dimensional images from computerised tomography (CT) or magnetic 
resonance imaging (MRI). AR can use a variety of display devices including monitors, smartphone screens and headmounted displays. In some instances, the combined image may be supplemented via different sensory modalities including auditory, haptic and somatosensory sensation.

MR, sometimes referred to as hybrid reality, is a concept which, within the reality-virtuality continuum, encompasses AR (1). MR is so named as it includes aspects of both the real and virtual worlds. Practically, MR as a technology is more advanced than AR. MR orthoscopically anchors virtual objects, such as three-dimensionally reconstructed CT or MRI scans, into the real world and allows the user to both visualise and interact with them. To experience MR, the user requires a head-mounted display with see-through capability which superimposes the image of the virtual object on to the real-world view. HoloLens 2, released by Microsoft (Redmond, Washington, USA) in 2019, is a well-known example of a publicly available MR device.

The strength of AR and MR lies in their ability to allow the user to more easily process real and virtual data simultaneously. AR and MR have so far shown the most potential in the engineering, education and entertainment industries. The use of AR and MR in surgery is a new and rapidly developing field, with research by clinicians of various surgical specialities showing that $A R$ and MR have many potential applications $(2,3)$. For example, it was reported that AR aided intraoperative localisation and resection of colorectal liver metastases (4). AR has been shown to help significantly reduce the radiation dosage to patients during percutaneous vertebroplasty (5). A reduction in operating time, as well as reduced pain at one-year follow-up, was seen with the use of MR in percutaneous kyphoplasty (6). Very recently, the use of MR during cardiac ablation procedures significantly improved the accuracy of intraprocedural navigation (7).

It is hoped that some surgeons will see their work transformed by AR and MR; this will require the continuing development of a strong evidence base. There is much ongoing research into the impact of this technology on areas including the safety of patients and clinicians, and the efficiency of healthcare services. Considering this, the primary objective of the proposed systematic review is to answer the following questions:

- What AR and MR technology platforms have been used intraoperatively? In what surgical contexts have AR and MR been used?

- What effects have AR and MR had on patient safety?

- During which procedures have AR and MR been used?

- What technical challenges and limitations have users of AR and MR faced?

- What effects have AR and MR had on healthcare costs?

The roll-out of AR and MR technologies into routine healthcare settings will only occur with high-quality evidence of cost-effective improvements to patient care. Through answering the above questions, this study aims to synthesise the current evidence base and provide directions for future research and development.

\section{Methods}

This systematic review protocol is reported in accordance with Preferred Reporting Items for Systematic Reviews and Meta-Analyses Protocols (PRISMA-P) guidelines (8), and is registered with International Prospective Register of Systematic Reviews (PROSPERO) (registration number CRD42020205892). The presented systematic literature search strategy has been designed a priori by authors E.B., B.L. and P.E.B.

\section{Data Sources}


A literature search will be performed using the electronic databases PubMed, Scopus, Cochrane Library (Cochrane Central Registry of Controlled Trials and Cochrane Database of Systematic Reviews), ClinicalTrials.gov and WHO International Clinical Trials Registry Platform. The keywords used are selected from key papers to create a broad search as described in Table 1. Search strings 1 and 2 will be combined using the Boolean term AND, then the following limits will be applied: (1) Publication date prior to the date of the search, (2) English language only. In addition, the reference lists of included articles will be searched.

Table 1

Literature search strategy: search strings 1 and 2 will be combined using the Boolean term AND then the limits will be applied

\section{Search Strategy}

1 = augmented reality OR mixed reality OR hybrid reality OR head-mounted display OR head-up display

2 = surgery OR surgeon OR surgica I OR intraoperative OR navigation OR visualisation OR imaging OR training OR safety

\section{Limits}

Publication date 1990 onwards; English language only.

\section{Study Selection and Analysis}

A broad search strategy will be used to identify all studies investigating the intraoperative use of AR or MR. Literature search results will be managed using Mendeley Desktop (Mendeley Desktop version 1.19.6, Elsevier, Amsterdam, Netherlands). Electronic citations, including available abstracts, will be independently screened by three reviewers (E.B., J.S. and M.S.). Discrepancies will be resolved by discussion or by referral to a fourth reviewer (B.L.). Prespecified inclusion criteria are that studies report empirical data on the use of AR or MR intraoperatively.

The following will be excluded: studies using AR or MR only in the context of simulation or training; studies describing only the technique of using AR or MR in the absence of clinical data; studies reporting the use of VR in the absence of AR or MR; protocol papers; systematic reviews; meta-analyses; review articles; individual opinions such as letters or commentaries; articles not published in the English language; and studies on animal models.

\section{Data Extraction and Analysis}

The data from all articles in the final review will be extracted by three independent authors (E.B., J.S. and M.S.) using a structured, standardised proforma (Table 2) to ensure consistency in the method of appraisal of these articles. This proforma has been designed a priori and tested on two articles. Any discrepancies will be resolved by consensus or by referral to a fourth reviewer (B.L.).

Table 2

Data extraction proforma

\begin{tabular}{|llllll|}
\hline Author Year & $\begin{array}{l}\text { Study } \\
\text { design }\end{array}$ & $\begin{array}{l}\text { No. of } \\
\text { participants }\end{array}$ & Technology Device Ergonomics Manufacturer Cost \\
\hline
\end{tabular}

\section{Table 2 continued}




\begin{tabular}{|llllllll|}
\hline $\begin{array}{l}\text { Source } \\
\text { of real } \\
\text { data }\end{array}$ & $\begin{array}{l}\text { Source } \\
\text { of } \\
\text { virtual } \\
\text { data }\end{array}$ & $\begin{array}{l}\text { Image-to- } \\
\text { patient } \\
\text { registration } \\
\text { technique }\end{array}$ & $\begin{array}{l}\text { Technical } \\
\text { challenges/ }\end{array}$ & $\begin{array}{l}\text { Elective or } \\
\text { emergency }\end{array}$ & $\begin{array}{l}\text { Adult } \\
\text { or } \\
\text { child }\end{array}$ & $\begin{array}{l}\text { Study context } \\
\text { (pre- vs } \\
\text { intraoperative) }\end{array}$ & $\begin{array}{l}\text { Speciality } \\
\text { of } \\
\text { operating } \\
\text { surgeon(s) }\end{array}$ \\
\hline
\end{tabular}

\section{Table 2 continued}

\begin{tabular}{|c|c|c|c|c|c|}
\hline $\begin{array}{l}\text { Operative } \\
\text { time }\end{array}$ & $\begin{array}{l}\text { Postoperative } \\
\text { complications }\end{array}$ & $\begin{array}{l}\text { Length of } \\
\text { inpatient stay }\end{array}$ & $\begin{array}{l}\text { User } \\
\text { satisfaction } \\
\text { score }\end{array}$ & $\begin{array}{l}\text { Other qualitative } \\
\text { outcomes }\end{array}$ & $\begin{array}{l}\text { Other quantitative } \\
\text { outcomes }\end{array}$ \\
\hline
\end{tabular}

\section{Risk of Bias Assessment}

Included articles will be critically appraised for methodological quality and risk of bias. The extent of discussion of individual articles in this review will be weighted according to each article's evidence level. For each objective of this review, sources of bias will be discussed collectively.

Observational cohort studies will be assessed using the Cochrane Collaboration Risk of Bias in Non-randomised Studies - of Interventions (ROBINS-I) Tool. This will evaluate the risk of bias due to confounding, selection, measurement, and interpretation. The risk of bias assessment will be presented using a traffic-light grid in the style recommended by the Cochrane Collaboration.

\section{Data synthesis}

A narrative synthesis will be performed. This will be structured according to the core questions of the review and subcategorised by device used. We will perform subgroup analysis based upon the available data, such as differences between surgical specialities and context.

If there are three or more studies with low risk of bias and data amenable to meta-analysis, then this will be performed. We will explore sources of potential clinical and methodological heterogeneity based on the study design, population, intervention and comparator characteristics and outcomes. Statistical heterogeneity will be assessed using the chisquare test and quantified with the $\mathrm{I}^{2}$ statistic. The thresholds for interpretation of $\mathrm{I}^{2}$ will be in accordance with the definitions presented in the Cochrane Handbook for Systematic Reviews of Interventions.

Data synthesis will be performed using SPSS Statistics (SPSS Statistics software version 27.0, International Business Machines Corporation (IBM), New York, US).

The quality of evidence will be rated using the Grading of Recommendations Assessment, Development and Evaluation (GRADE) approach. The GRADE analysis will be performed as per the Cochrane Handbook for Systematic Reviews of Interventions and applied for all outcomes. All studies will be ranked based on the level of evidence and graded based on the quality of evidence by three authors independently. Data will be presented in a summary of findings table.

\section{Discussion}


AR and MR technologies have the potential to revolutionise surgical interventions, leading to a step-change in surgical precision and patient outcomes, however, the difference between the technology required for such a future, and the technology currently available, remains unclear. In order for surgeons to better understand the use cases, benefits, and limitations of these technologies, an up-to-date synthesis of the literature is required.

\section{List Of Abbreviations}

PROSPERO: International Prospective Register of Systematic Reviews; AR: augmented reality; MR: mixed reality; VR: virtual reality; CT: computerised tomography; MRI: magnetic resonance imaging; PRISMA-P: Preferred Reporting Items for Systematic reviews and Meta-Analysis Protocols; PRISMA: Preferred Reporting Items for Systematic reviews and Meta-Analysis; WHO: World Health Organisation; ROBINS-I: Risk of Bias in Non-randomised Studies - of Interventions; STROBE: STrengthening the Reporting of OBservational studies in Epidemiology; GRADE: Grading of Recommendations Assessment, Development and Evaluation

\section{Declarations}

\section{Ethics approval}

Not applicable.

\section{Consent for publication}

Not applicable.

\section{Availability of data and materials}

Not applicable.

\section{Competing interests}

None.

\section{Funding}

None.

\section{Authors' contributions}

E.B. - concept, protocol design, manuscript preparation.

J.S. - manuscript preparation.

M.S. - manuscript preparation.

B.L. - concept, protocol design, manuscript revision. 


\section{Acknowledgements}

None.

\section{References}

1. Milgram P, Kishimo F. A taxonomy of mixed reality. IEICE Trans Inf Syst. 1994;77(12):1321-9.

2. Fida B, Cutolo F, di Franco G, Ferrari M, Ferrari V. Augmented reality in open surgery. Updates Surg [Internet]. 2018;70(3):389-400. Available from: https://doi.org/10.1007/s13304-018-0567-8

3. Vávra P, Roman J, Zonča P, Ihnát P, Němec M, Kumar J, et al. Recent Development of Augmented Reality in Surgery: A Review. J Healthc Eng. 2017;2017.

4. Ntourakis D, Memeo R, Soler L, Marescaux J, Mutter D, Pessaux P. Augmented Reality Guidance for the Resection of Missing Colorectal Liver Metastases: An Initial Experience. World J Surg. 2016;40(2):419-26.

5. Auloge P, Cazzato RL, Ramamurthy N, de Marini P, Rousseau C, Garnon J, et al. Augmented reality and artificial intelligence-based navigation during percutaneous vertebroplasty: a pilot randomised clinical trial. Eur Spine J [Internet]. 2020;29(7):1580-9. Available from: https://doi.org/10.1007/s00586-019-06054-6

6. Wei P, Yao Q, Xu Y, Zhang H, Gu Y, Wang L. Percutaneous kyphoplasty assisted with/without mixed reality technology in treatment of OVCF with IVC: A prospective study. J Orthop Surg Res. 2019;14(1):1-9.

7. Avari Silva JN, Southworth MK, Blume WM, Andrews C, Van Hare GF, Dalal AS, et al. First-In-Human Use of a Mixed Reality Display During Cardiac Ablation Procedures. JACC Clin Electrophysiol [Internet]. 2020;6(8):1023-5. Available from: https://doi.org/10.1016/j.jacep.2020.04.036

8. Shamseer L, Moher D, Clarke M, Ghersi D, Liberati A, Petticrew M, Shekelle P SL. Prisma-P 2015. BMJ Br Med J. 2015;

\section{Supplementary Files}

This is a list of supplementary files associated with this preprint. Click to download.

- PRISMAPchecklist.docx

- PRISMAPchecklist.docx 